\title{
INTEGRATION FOR SPECIAL THIRD-ORDER ORDINARY DIFFERENTIAL EQUATIONS USING IMPROVED RUNGE-KUTTA DIRECT METHOD
}

\author{
Kasim Abbas Hussain ${ }^{1,2^{*}}$, Fudziah Ismail ${ }^{1,3}$, Norazak Senu ${ }^{1,3}$, Faranak Rabiei ${ }^{1,3}$ \\ and Rabha Ibrahim ${ }^{4}$
}

${ }^{1}$ Department of Mathematics, Faculty of Science, Universiti Putra Malaysia, 43400 Serdang, Selangor

${ }^{2}$ Department of Mathematics, Collage of Science, Al-Mustansiriyah University, Baghdad, Iraq

${ }^{3}$ Institute for Mathematical Research, Universiti Putra Malaysia, 43400 Serdang, Selangor

${ }^{4}$ Center of Mobile Cloud Computing Research, Faculty of Computer Science and Information Technology, University of Malaya, 50603 Kuala Lumpur

*Corresponding Author: Email : kasimmath2011@yahoo.com

\begin{abstract}
In this paper, we derive an explicit four stage fifth-order Improved Runge-Kutta (IRKD) method for numerical integration of special third-order ordinary differential equation. The method proposed here is two-step in nature and require less number of stages per step compared with the existing Runge-Kutta (RK) method. The stability polynomial of the IRKD method is presented. Numerical results are given to illustrate the efficiency of the proposed method compared to the RK method and direct Runge-Kutta (RKD) method for solving special third-order ordinary differential equations.
\end{abstract}

(Keywords: Special third-order ordinary differential equations, Runge-Kutta method, IRKD method, Stability Ploynomial)

\section{INTRODUCTION}

In this article, we are concerned with the special thirdorder ordinary differential equations (ODEs), which is defined as follows:

with initial conditions

$$
y^{\prime \prime \prime}(x)=f(x, y)
$$

$$
\left.y\left(x_{0}\right)=\eta_{0}, y^{\prime}\left(x_{0}\right)=\eta_{0}^{\prime}, y^{\prime \prime}\left(x_{0}\right)\right)=\eta_{0}^{\prime \prime} .
$$

where $f: R \times R \rightarrow R$ is a continuous valued function which does not contain the first derivative $y^{\prime}(x)$ and second derivative $y^{\prime \prime}(x)$. This type of problems often arises in a variety of applied sciences and engineering such as physics, molecular dynamics, the motion of rocket, biology and chemistry. Traditionally, most researchers solve the third-order ODE by transforming it to a system of the first order ordinary differential equations ODEs, then solve it using a suitable numerical method (see $[10,11,12,13]$ ). However, Several researchers (see [3, 4]) observed the drawback of this technique whereby it wasted a lot of computing time and human effort. Therefore, direct integration methods have attracted significant attention from several authors for solving third-order ODEs. For instance, Awoyemi [1] constructed the P-stable multistep collocation method to solve directly thirdorder ODEs using constant step size. Suleiman [8] presented a direct integration method to solve higher order ODEs using variable step size codes. Awoyemi and Idowu [2] derived the hybrid collocation method for solving third order ordinary differential equations. Waeleh et al. [5] presented the two point block method to to solve third-order ODEs directly. Majid et al. [7] introduced a block implicit method for directly solving third-order ODEs using variable step size codes. This paper primarily aims to derive a new improved RungeKutta method, namely IRKD method to solve special third-order ODEs directly.

\section{THE GENERAL FORM OF IRKD METHOD}

Motivated by the technique in Rabiei [9] for obtaining the algebraic order equations for Runge-Kutta Nyström method, we derive the algebraic order conditions for the IRKD method for solving special third-order ODEs directly. Consequently, the general $s$-stages IRKD method for solving (1) can be defined as follows: 


$$
\begin{aligned}
& y_{n+1}=y_{n}+\frac{3}{2} h y_{n}^{\prime}-\frac{1}{2} h y_{n-1}^{\prime}+\frac{5}{12} h^{2} y_{n}^{\prime \prime} \\
& -\frac{5}{12} h^{2} y_{n-1}^{\prime \prime}+h^{3} \sum_{i=2}^{s} b_{i}^{\prime \prime}\left(k_{i}-k_{-i}\right), \\
& y_{n+1}^{\prime}=y_{n}^{\prime}+\frac{3}{2} h y_{n}^{\prime \prime}-\frac{1}{2} h y_{n-1}^{\prime \prime}+h^{2} \sum_{i=2}^{S} b_{i}^{\prime}\left(k_{i}-k_{-i}\right) \text {, } \\
& y_{n+1}^{\prime \prime}=y_{n}^{\prime \prime}+h\left(b_{-1} k_{-1}+b_{1} k_{1}+\sum_{i=2}^{S} b_{i}\left(k_{i}-k_{-i}\right)\right) \text {, } \\
& y_{n+1}=y_{n}+\frac{3}{2} h y_{n}^{\prime}-\frac{1}{2} y_{n-1}^{\prime}+\frac{5}{12} h^{2} y_{n}^{\prime \prime}-\frac{5}{12} h^{2} y_{n-1}^{\prime \prime} \\
& +h^{3}\left(b_{2}^{\prime \prime}\left(k_{2}-k_{-2}\right)+b_{3}^{\prime \prime}\left(k_{3}-k_{-3}\right)\right. \\
& \left.+b_{4}^{\prime \prime}\left(k_{4}-k_{-4}\right)\right) \\
& y_{n+1}^{\prime}=y_{n}^{\prime}+\frac{3}{2} h y_{n}^{\prime \prime}-\frac{1}{2} h y_{n-1}^{\prime \prime}+h^{2}\left(b_{2}^{\prime}\left(k_{2}-k_{-2}\right)\right. \\
& \left.+b_{3}^{\prime}\left(k_{3}-k_{-3}\right)+b_{4}^{\prime}\left(k_{4}-k_{-4}\right)\right), \\
& y_{n+1}^{\prime \prime}=y_{n}^{\prime \prime}+h\left(b_{-1} k_{-1}+b_{1} k_{1}+b_{2}\left(k_{2}-k_{-2}\right)\right. \\
& \left.+b_{3}\left(k_{3}-k_{-3}\right)+b_{4}\left(k_{4}-k_{-4}\right)\right) \text {, }
\end{aligned}
$$

$$
\begin{aligned}
& k_{1}=f\left(x_{n}, y_{n}\right), \\
& k_{-1}=f\left(x_{n-1}, y_{n-1}\right), \\
& k_{i}=f\left(x_{n}+c_{i} h, y_{n}+h c_{i} y_{n}^{\prime}+\frac{1}{2} h^{2} c_{i}^{2} y_{n}^{\prime \prime}+\right. \\
& \left.\quad+h^{3} \sum_{j=1}^{s} a_{i j} k_{j}\right) \\
& k_{-i}=f\left(x_{n-1}+c_{i} h, y_{n-1}+h c_{i}+\frac{1}{2} h^{2} c_{i}^{2} y_{n-1}^{\prime \prime}\right. \\
& \left.+h^{3} \sum_{j=1}^{s} a_{i j} k_{-j}\right) .
\end{aligned}
$$

All coefficients $b_{i}, b_{i}^{\prime}, b_{i}^{\prime \prime}, a_{i j}$, and $c_{i}$ of the IRKD method are supposed to be real and for $i=2,, \ldots, s$ and $j=1,2, \ldots, s$.

The IRKD method expression (2)-(8) is extended using Taylor series expansion to obtain the coefficients of the IRKD method. After doing some algebraic simplifications, this expansion is equated to the true solution which is presented by the Taylor series expansion. As a result, the system of nonlinear algebraic equations is obtained, which is denoted as order conditions. The algebraic and numerical calculations are carried out using the Maple package. The IRKD method can be represented in Butcher notation as follows:

\begin{tabular}{c|c}
$c$ & $A$ \\
\hline$b_{-1}$ & $b^{T}$ \\
& $b^{\prime T}$ \\
& $b^{\prime \prime T}$
\end{tabular}

\section{DERIVATION OF FIFTH-ORDER IRKD METHOD WITH FOUR STAGE}

The general form of four-stage fifth-order IRKD method has the following form

where

$$
\begin{aligned}
& k_{1}=f\left(x_{n}, y_{n}\right), \\
& k_{-1}=f\left(x_{n-1}, y_{n-1}\right), \\
& k_{2}=f\left(x_{n}+c_{2} h, y_{n}+h c_{2} y_{n}^{\prime}+\frac{1}{2} h^{2} c_{2}^{2} y_{n}^{\prime \prime}\right. \\
& +h^{3}\left(a_{21} k_{1}\right) \\
& k_{-2}=f\left(x_{n-1}+c_{2} h, y_{n-1}+h c_{2} y_{n-1}^{\prime}+\frac{1}{2} h^{2} c_{2}^{2} y_{n-1}^{\prime \prime}\right. \\
& +h^{3}\left(a_{21} k_{-1}\right) \\
& k_{3}=f\left(x_{n}+c_{3} h, y_{n}+h c_{3} y_{n}^{\prime}+\frac{1}{2} h^{2} c_{3}^{2} y_{n}^{\prime \prime}\right. \\
& \left.+h^{3}\left(a_{31} k_{1}+a_{32} k_{2}\right)\right), \\
& k_{-3}=f\left(x_{n-1}+c_{3} h, y_{n-1}+h c_{3} y_{n-1}^{\prime}+\frac{1}{2} h^{2} c_{3}^{2} y_{n-1}^{\prime \prime}\right. \\
& \left.+h^{3}\left(a_{31} k_{-1}+a_{32} k_{-2}\right)\right), \\
& k_{4}=f\left(x_{n}+c_{4} h, y_{n}+h c_{4} y_{n}^{\prime}+\frac{1}{2} h^{2} c_{4}^{2} y_{n}^{\prime \prime}\right. \\
& \left.+h^{3}\left(a_{41} k_{1}+a_{42} k_{2}+a_{43} k_{3}\right)\right), \\
& k_{-4}=f\left(x_{n-1}+c_{4} h, y_{n-1}+h c_{4} y_{n-1}^{\prime}+\frac{1}{2} h^{2} c_{4}^{2} y_{n-1}^{\prime \prime}\right. \\
& \left.+h^{3}\left(a_{41} k_{-1}+a_{42} k_{-2}+a_{43} k_{-3}\right)\right) .
\end{aligned}
$$

By applying the Taylor series expansion on IRKD method (9) - (17) we obtain the order conditions up to order six as follows:

The order conditions for $y$ :

order 4:

$$
\sum_{i=2}^{4} b_{i}^{\prime \prime}=\frac{1}{6},
$$


order 5:

$$
\sum_{i=2}^{4} b_{i}^{\prime \prime} c_{i}=\frac{31}{720}
$$

order 6:

$$
\sum_{i=2}^{4} b_{i}^{\prime \prime} c_{i}^{2}=\frac{1}{60} .
$$

The order conditions for $y^{\prime}$ :

order 3:

$$
\sum_{i=2}^{4} b_{i}^{\prime}=\frac{5}{12}
$$

order 4:

$$
\sum_{i=2}^{4} b_{i}^{\prime} c_{i}=\frac{1}{6}
$$

order 5:

$$
\sum_{i=2}^{4} b_{i}^{\prime} c_{i}^{2}=\frac{31}{360},
$$

order 6:

$$
\begin{aligned}
& \sum_{i=2}^{4} b_{i}^{\prime} c_{i}^{3}=\frac{1}{20}, \\
& \sum_{i=2}^{4} b_{i}^{\prime} a_{i j}=\frac{1}{120} .
\end{aligned}
$$

The order conditions for $y^{\prime \prime}$ :

order 1:

$$
b_{1}-b_{-1}=1 \text {, }
$$

order 2:

$$
b_{-1}+\sum_{i=2}^{4} b_{i}=\frac{1}{2},
$$

order 3:

$$
\sum_{i=2}^{4} b_{i} c_{i}=\frac{5}{12},
$$

order 4:

$$
\sum_{i=2}^{4} b_{i} c_{i}^{2}=\frac{1}{3}
$$

order 5:

$$
\sum_{i=2}^{4} b_{i} c_{i}^{3}=\frac{31}{120}
$$

$$
\sum_{i=2}^{4} b_{i} a_{i j}=\frac{31}{720},
$$

order 6:

$$
\begin{aligned}
\sum_{i=2}^{4} b_{i} c_{i}^{4}=\frac{1}{5} & \\
\sum_{i=2}^{4} b_{i} a_{i j} c_{j} & =\frac{1}{120}, \quad \sum_{i=2}^{4} b_{i} c_{i} a_{i j}=\frac{1}{30} .
\end{aligned}
$$

To construct a four-stage fifth-order IRKD method, the order conditions up to order five (18)-(19), (21)-(23) and (26)-(31), which include 11 nonlinear equations with 20 unknowns need to be solved. Therefore, the resulting system has nine free parameters; setting $c_{2}=\frac{1}{12}, c_{3}=\frac{2}{9}, c_{4}=\frac{2}{3}, a_{32}=\frac{1}{32} \quad$ and $\quad b_{2}^{\prime \prime}=\frac{1}{20}$, solving the system simultaneously yields a solution as follows:

$$
\begin{aligned}
& b_{1}=-\frac{27}{40}, b_{2}=\frac{88}{25}, b_{3}=-\frac{459}{200}, b_{4}=\frac{19}{20}, b_{-1}=-\frac{67}{40}, \\
& b_{2}^{\prime}=-\frac{2}{525}, b_{3}^{\prime}=\frac{51}{200}, b_{4}^{\prime}=\frac{139}{840}, b_{3}^{\prime \prime}=\frac{7}{80}, b_{4}^{\prime \prime}=\frac{7}{240} \\
& a_{42}=-\frac{352}{95} a_{21}+\frac{459}{190} a_{31}+\frac{6611}{54720}-a_{41}-a_{43} .
\end{aligned}
$$

As a results, we have four free parameters $a_{21}, a_{31}, a_{41}$ and $a_{43}$ which can be chosen by minimizing the error equations. According to Dormand et al. [14], the free parameters can be selected by minimizing the global error of the sixth-order conditions. The approach is performed as follows:

Firstly: the error equations of $y, y^{\prime}$ and $y^{\prime \prime}$ is determined respectively as follows:

$\left\|\tau^{\prime \prime(6)}\right\|_{2}=\sqrt{\sum_{i=1}^{n_{p}^{\prime \prime}+1}\left(\tau_{i}^{\prime \prime(6)}\right)^{2}}$,

$\left\|\tau^{\prime(6)}\right\|_{2}=\sqrt{\sum_{i=1}^{n_{p}^{\prime}+1}\left(\tau_{i}^{\prime(6)}\right)^{2}}$,

$\left\|\tau^{(6)}\right\|_{2}=\sqrt{\sum_{i=1}^{n_{p}+1}\left(\tau_{i}^{(6)}\right)^{2}}$.

Secondly: The global error norm is determined as follows:

$\left\|\tau_{g}^{(6)}\right\|_{2}=$

$\sqrt{\sum_{i=1}^{n_{p}^{\prime \prime}+1}\left(\tau_{i}^{\prime \prime(6)}\right)^{2}+\sum_{i=1}^{n_{p}^{\prime}+1}\left(\tau_{i}^{\prime(6)}\right)^{2}+\sum_{i=1}^{n_{p}+1}\left(\tau_{i}^{(6)}\right)^{2}}$

Finally: minimize the global error in equation (37) with respect to the free parameters $a_{21}, a_{31}, a_{41}$ and $a_{43}$, 
Therefore, we obtain the free parameters as follows:

$a_{21}=-\frac{367}{120344}, a_{31}=-\frac{13703}{417136}, a_{41}=\frac{20457}{17918}$

and $a_{43}=\frac{34752}{45617}$. Which leads to $a_{42}=-\frac{41509}{22428}$ and then the error coefficients for $y, y^{\prime}$ and $y^{\prime \prime}$ are computed respectively as follows:

$$
\begin{aligned}
& \left\|\tau^{\prime \prime(6)}\right\|_{2}=9.645061728 \times 10^{-4}, \\
& \left\|\tau^{\prime(6)}\right\|_{2}=1.826131687 \times 10^{-3}, \\
& \left\|\tau^{(6)}\right\|_{2}=1.777263374 \times 10^{-2},
\end{aligned}
$$

and the global error norm is

$\left\|\tau_{g}^{(6)}\right\|_{2}=1.789222008 \times 10^{-2}$.

where $\tau^{\prime \prime(6)}, \tau^{\prime(6)}$ and $\tau^{(6)}$ are the error equations of sixth-order conditions for $y, y^{\prime}$ and $y^{\prime \prime}$ respectively. Finally, the fifth-order IRKD method with four-stage are written in Butcher tableau and denoted as IRKD5 method as follows:

Table 1. Butcher Tableau of IRKD5 Method

\begin{tabular}{c|cccc}
$\frac{1}{12}$ & $-\frac{367}{120344}$ & & & \\
$\frac{2}{9}$ & $-\frac{13703}{417136}$ & $\frac{1}{32}$ & & \\
$\frac{2}{3}$ & $\frac{20457}{17918}$ & $-\frac{41509}{22428}$ & $\frac{34752}{45617}$ & \\
\hline$-\frac{67}{40}$ & $-\frac{27}{40}$ & $\frac{88}{25}$ & $-\frac{459}{200}$ & $\frac{19}{20}$ \\
& & $-\frac{2}{525}$ & $\frac{51}{200}$ & $\frac{139}{840}$ \\
& $\frac{1}{20}$ & $\frac{7}{80}$ & $\frac{7}{240}$
\end{tabular}

\section{STABILITY POLYNOMIAL OF IRKD METHOD}

To obtain the stability polynomial of the IRKD method, we can rewrite the IRKD method as follows:

$$
\begin{aligned}
y_{n+1}= & y_{n}+\frac{3}{2} h y_{n}^{\prime}-\frac{1}{2} h y_{n-1}^{\prime}+\frac{5}{12} h^{2} y_{n}^{\prime \prime}-\frac{5}{12} h^{2} y_{n-1}^{\prime \prime} \\
& +h^{3} \sum_{i=2}^{s} b_{i}^{\prime \prime}\left(f\left(x_{n}+c_{i} h, Y_{i}\right)-\right.
\end{aligned}
$$

$$
\left.f\left(x_{n-1}+c_{i} h, Y_{-i}\right)\right)
$$

$$
\begin{aligned}
y_{n+1}^{\prime}= & y_{n}^{\prime}+\frac{3}{2} h y_{n}^{\prime \prime}-\frac{1}{2} h y_{n-1}^{\prime \prime} \\
& +h^{2} \sum_{i=2}^{s} b_{i}^{\prime}\left(f\left(x_{n}+c_{i} h, Y_{i}\right)-\right. \\
& \left.f\left(x_{n-1}+c_{i} h, Y_{-i}\right)\right), \\
y_{n+1}^{\prime \prime}= & y_{n}^{\prime \prime}+h\left(b_{-1} Y_{-1}+b_{1} Y_{1}\right. \\
+ & h^{2} \sum_{i=2}^{s} b_{i}\left(f\left(x_{n}+c_{i} h, Y_{i}\right)-\right. \\
& \left.f\left(x_{n-1}+c_{i} h, Y_{-i}\right)\right),
\end{aligned}
$$

where

$$
\begin{aligned}
& Y_{1}=y_{n}, \\
& Y_{-1}=y_{n-1}, \\
& Y_{i}=y_{n}+h c_{i} y_{n}^{\prime}+\frac{1}{2} h^{2} c_{i}^{2} y_{n}^{\prime \prime} \\
& \quad+h^{3} \sum_{j=1}^{i-1} a_{i j}\left(f\left(x_{n}+c_{i} h, Y_{i}\right)\right. \\
& Y_{-i}=y_{n-1}+h c_{i} y_{n-1}^{\prime}+\frac{1}{2} h^{2} c_{i}^{2} y_{n-1}^{\prime \prime} \\
& \quad+h^{3} \sum_{j=1}^{i-1} a_{i j}\left(f\left(x_{n-1}+c_{i} h, Y_{-i}\right) .\right.
\end{aligned}
$$

for $i=2,3, \ldots, s$.

We consider the following test equation

$$
y^{\prime \prime \prime}=-\lambda^{3} y
$$

Now, applying the IRKD method (38)-(44) to the test equation (45), which leads to

$$
\begin{aligned}
& y_{n+1}=y_{n}+\frac{3}{2} h y_{n}^{\prime}-\frac{1}{2} h y_{n-1}^{\prime}+\frac{5}{12} h^{2} y_{n}^{\prime \prime}-\frac{5}{12} h^{2} y_{n-1}^{\prime \prime} \\
&+h^{3} \sum_{i=2}^{s}-\lambda^{3} b_{i}^{\prime \prime}\left(Y_{i}-Y_{-i}\right) \\
& y_{n+1}^{\prime}=y_{n}^{\prime}+\frac{3}{2} h y_{n}^{\prime \prime}-\frac{1}{2} h y_{n-1}^{\prime \prime} \\
&+ h^{2} \sum_{i=2}^{s}-\lambda^{3} b_{i}^{\prime}\left(Y_{i}-Y_{-i}\right) \\
& y_{n+1}^{\prime \prime}=y_{n}^{\prime \prime}+h\left(\left(-\lambda^{3} b_{-1}\right) Y_{-1}+\left(-\lambda^{3} b_{1}\right) Y_{1}\right. \\
&\left.+\sum_{i=2}^{s}-\lambda^{3} b_{i}\left(Y_{i}-Y_{-i}\right)\right)
\end{aligned}
$$

where

$$
\begin{aligned}
& Y_{i}=y_{n}+h c_{i} y_{n}^{\prime}+\frac{1}{2} h^{2} c_{i}^{2} y_{n}^{\prime \prime}+h^{3} \sum_{j=1}^{i-1}-\lambda^{3} a_{i j} Y_{i} \\
& Y_{-i}=y_{n-1}+h c_{i} y_{n-1}^{\prime}+\frac{1}{2} h^{2} c_{i}^{2} y_{n-1}^{\prime \prime} \\
&+h^{3} \sum_{j=1}^{i-1}-\lambda^{3} a_{i j} Y_{-i}
\end{aligned}
$$


Malaysian Journal of Science 34 (2) : 172-179 (2015)

Multiplying equations (47) by $h$ and (48) by $h^{2}$, we get

$$
\begin{aligned}
& h y_{n+1}^{\prime}=h y_{n}^{\prime}+\frac{3}{2} h^{2} y_{n}^{\prime \prime}-\frac{1}{2} h^{2} y_{n-1}^{\prime \prime}+\left(-\lambda^{3} h^{3}\right) \sum_{i=2}^{s} b_{i}^{\prime}\left(Y_{i}-Y_{-i}\right) \\
& h^{2} y_{n+1}^{\prime \prime}=h^{2} y_{n}^{\prime \prime}+\left(-\lambda^{3} h^{3}\right)\left(b_{-1} Y_{-1}+b_{1} Y_{1}+\sum_{i=2}^{s} b_{i}\left(Y_{i}-Y_{-i}\right)\right)
\end{aligned}
$$

The equations (46), (51) and (52) can be represented in the following matrix format

$$
\left[\begin{array}{c}
y_{n+1} \\
y_{n} \\
h y_{n+1}^{\prime} \\
h y_{n}^{\prime} \\
h^{2} y_{n+1}^{\prime \prime} \\
h^{2} y_{n}^{\prime \prime}
\end{array}\right]=\left[\begin{array}{rrrrrr}
1 & 0 & \frac{3}{2} & -\frac{1}{2} & \frac{5}{12} & -\frac{5}{12} \\
0 & 0 & 0 & 0 & 0 & 0 \\
0 & 0 & 1 & 0 & \frac{3}{2} & -\frac{1}{2} \\
0 & 0 & 0 & 1 & 0 & 0 \\
0 & 0 & 0 & 0 & 1 & 0 \\
0 & 0 & 0 & 0 & 0 & 1
\end{array}\right]\left[\begin{array}{c}
y_{n} \\
y_{n-1} \\
h y_{n}^{\prime} \\
h y_{n-1}^{\prime} \\
h^{2} y_{n}^{\prime \prime} \\
h^{2} y_{n-1}^{\prime \prime}
\end{array}\right]+\left(-\lambda^{3} h^{3}\right)\left[\begin{array}{cccccc}
0 & 0 & b_{2}^{\prime \prime} & b_{3}^{\prime \prime} & \cdots & b_{s}^{\prime \prime} \\
0 & 0 & 0 & 0 & \cdots & 0 \\
0 & 0 & b_{2}^{\prime} & b_{3}^{\prime} & \cdots & b_{s}^{\prime} \\
0 & 0 & 0 & 0 & \cdots & 0 \\
b_{-1} & b_{1} & b_{2} & b_{3} & \cdots & b_{s} \\
0 & 0 & 0 & 0 & 0 & 0
\end{array}\right]\left(\left[\begin{array}{c}
0 \\
Y_{1} \\
Y_{2} \\
\vdots \\
Y_{s}
\end{array}\right]-\left[\begin{array}{c}
Y_{-1} \\
0 \\
Y_{-2} \\
\vdots \\
Y_{-s}
\end{array}\right]\right)
$$

Also, the matrix format of equations (49) and (50) is defined as

$$
\left[\begin{array}{c}
0 \\
Y_{1} \\
Y_{2} \\
\vdots \\
Y_{S}
\end{array}\right]=\left[\begin{array}{cccccc}
0 & 0 & 0 & 0 & 0 & 0 \\
1 & 0 & 0 & 0 & 0 & 0 \\
1 & 0 & c_{2} & 0 & \frac{1}{2} c_{2}^{2} & 0 \\
1 & 0 & c_{3} & 0 & \frac{1}{2} c_{3}^{2} & 0 \\
\vdots & \vdots & \vdots & \vdots & \vdots & \vdots \\
1 & 0 & c_{s} & 0 & \frac{1}{2} c_{s}^{2} & 0
\end{array}\right]\left[\begin{array}{c}
y_{n} \\
y_{n-1} \\
h y_{n}^{\prime} \\
h y_{n-1}^{\prime} \\
h^{2} y_{n}^{\prime \prime} \\
h^{2} y_{n-1}^{\prime \prime}
\end{array}\right]+\left(-\lambda^{3} h^{3}\right)\left[\begin{array}{cccccc}
1 & 0 & 0 & \ldots & 0 & 0 \\
0 & 0 & 0 & \ldots & 0 & 0 \\
a_{21} & a_{21} & 0 & \ldots & 0 & 0 \\
a_{31} & a_{31} & a_{32} & \ldots & 0 & 0 \\
\vdots & \vdots & \vdots & \ddots & \vdots & \vdots \\
a_{s 1} & a_{s 1} & a_{s 2} & \ldots & a_{s s-1} & 0
\end{array}\right]\left[\begin{array}{c}
0 \\
Y_{1} \\
Y_{2} \\
\vdots \\
Y_{s}
\end{array}\right]
$$

and

$$
\left[\begin{array}{c}
0 \\
Y_{-1} \\
Y_{-2} \\
\vdots \\
Y_{-s}
\end{array}\right]=\left[\begin{array}{cccccc}
0 & 1 & 0 & 0 & 0 & 0 \\
0 & 0 & 0 & 0 & 0 & 0 \\
0 & 1 & 0 & c_{2} & 0 & \frac{1}{2} c_{2}^{2} \\
0 & 1 & 0 & c_{3} & 0 & \frac{1}{2} c_{3}^{2} \\
\vdots & \vdots & \vdots & \vdots & \vdots & \vdots \\
0 & 1 & 0 & c_{s} & 0 & \frac{1}{2} c_{s}^{2}
\end{array}\right]\left[\begin{array}{c}
y_{n} \\
y_{n-1} \\
h y_{n}^{\prime} \\
h y_{n-1}^{\prime} \\
h^{2} y_{n}^{\prime \prime} \\
h^{2} y_{n-1}^{\prime \prime}
\end{array}\right]+\left(-\lambda^{3} h^{3}\right)\left[\begin{array}{cccccc}
1 & 0 & 0 & \ldots & 0 & 0 \\
0 & 0 & 0 & \ldots & 0 & 0 \\
a_{21} & a_{21} & 0 & \ldots & 0 & 0 \\
a_{31} & a_{31} & a_{32} & \ldots & 0 & 0 \\
\vdots & \vdots & \vdots & \ddots & \vdots & \vdots \\
a_{s 1} & a_{s 1} & a_{s 2} & \ldots & a_{s s-1} & 0
\end{array}\right]\left[\begin{array}{c}
0 \\
Y_{-1} \\
Y_{-2} \\
\vdots \\
Y_{-s}
\end{array}\right]
$$

After carrying out some algebraic manipulation, we obtain the final matrix as follows

$$
\begin{aligned}
& u_{n+1}=P u_{n}+H B Y, \\
& Y=C u_{n}+H A Y .
\end{aligned}
$$

where

$$
A=\left[\begin{array}{cccccc}
1 & 0 & 0 & \cdots & 0 & 0 \\
0 & 0 & 0 & \cdots & 0 & 0 \\
a_{21} & a_{21} & 0 & \cdots & 0 & 0 \\
a_{31} & a_{31} & a_{32} & \cdots & 0 & 0 \\
\vdots & \vdots & \vdots & \ddots & \vdots & \vdots \\
a_{s 1} & a_{s 1} & a_{s 2} & \cdots & a_{s s-1} & 0
\end{array}\right], \quad B=\left[\begin{array}{cccccc}
0 & 0 & b_{2}^{\prime \prime} & b_{3}^{\prime \prime} & \cdots & b_{s}^{\prime \prime} \\
0 & 0 & 0 & 0 & \cdots & 0 \\
0 & 0 & b_{2}^{\prime} & b_{3}^{\prime} & \cdots & b_{s}^{\prime} \\
0 & 0 & 0 & 0 & \cdots & 0 \\
b_{-1} & b_{1} & b_{2} & b_{3} & \cdots & b_{s} \\
0 & 0 & 0 & 0 & 0 & 0
\end{array}\right] \quad \text { and }
$$




$$
P=\left[\begin{array}{rrrrrr}
1 & 0 & \frac{3}{2} & -\frac{1}{2} & \frac{5}{12} & -\frac{5}{12} \\
0 & 0 & 0 & 0 & 0 & 0 \\
0 & 0 & 1 & 0 & \frac{3}{2} & -\frac{1}{2} \\
0 & 0 & 0 & 1 & 0 & 0 \\
0 & 0 & 0 & 0 & 1 & 0 \\
0 & 0 & 0 & 0 & 0 & 1
\end{array}\right]
$$

Simplifying (57) we have,

$$
Y=(I-H A)^{-1} C u_{n}
$$$$
W(H)=\left[\begin{array}{cccccc}
1+H b^{\prime \prime T} P^{-1} C_{1} & H b^{\prime \prime T} P^{-1} C_{2} & \frac{3}{2}+H b^{\prime \prime T} P^{-1} C_{3} & -\frac{1}{2}+H b^{\prime \prime T} P^{-1} C_{4} & \frac{5}{12}+H b^{\prime \prime T} P^{-1} C_{5} & -\frac{5}{12}+H b^{\prime \prime T} P^{-1} C_{6} \\
1 & 0 & 0 & 0 & 0 & 0 \\
H b^{\prime T} P^{-1} C_{1} & H b^{\prime T} P^{-1} C_{2} & 1+H b^{\prime T} P^{-1} C_{3} & H b^{\prime T} P^{-1} C_{4} & \frac{3}{2}+H b^{\prime T} P^{-1} C_{5} & -\frac{1}{2}+H b^{\prime T} P^{-1} C_{6} \\
0 & 0 & 1 & 0 & 0 & 0 \\
H b^{T} P^{-1} C_{1} & H b^{T} P^{-1} C_{2} & H b^{T} P^{-1} C_{3} & H b^{T} P^{-1} C_{4} & 1+H b^{T} P^{-1} C_{5} & H b^{T} P^{-1} C_{6} \\
0 & 0 & 0 & 0 & 1 & 0
\end{array}\right]
$$

where $I$ is the identity matrix. Finally, substituting (58) into (56), we get

$$
u_{n+1}=\left(P+H B\left((I-H A)^{-1} C\right)\right) u_{n} .
$$

Therefore, equation (59) can be written as

$$
u_{n+1}=W(H) u_{n}
$$

where

Also where

$$
\begin{aligned}
& P^{-1}=(I-H A)^{-1}, \quad b^{\prime \prime}=\left[\begin{array}{llllll}
0 & 0 & b_{2}^{\prime \prime} & \ldots & b_{s}^{\prime \prime}
\end{array}\right]^{T}, \quad b^{\prime}=\left[\begin{array}{llllll}
0 & 0 & b_{2}^{\prime} & \ldots & b_{s}^{\prime}
\end{array}\right]^{T}, \quad b=\left[\begin{array}{llllll}
0 & 0 & b_{2} & \ldots & b_{s}
\end{array}\right]^{T}, \\
& C_{1}=\left[\begin{array}{lllll}
0 & 1 & 1 & \ldots & 1
\end{array}\right]^{T}, \quad C_{2}=\left[\begin{array}{lllllll}
-1 & 0 & -1 & \ldots & -1
\end{array}\right]^{T}, \quad C_{3}=\left[\begin{array}{llllll}
0 & 0 & c_{2} & \ldots & c_{s}
\end{array}\right]^{T}, \\
& C_{4}=\left[\begin{array}{lllllllll}
0 & 0 & -c_{2} & \ldots & -C_{s}
\end{array}\right]^{T}, \quad C_{5}=\left[\begin{array}{lllllll}
0 & 0 & \frac{1}{2} c_{2}^{2} & \ldots & \frac{1}{2} c_{s}^{2}
\end{array}\right]^{T}, \quad C_{6}=\left[\begin{array}{llllll}
0 & 0 & -\frac{1}{2} c_{2}^{2} & \ldots & -\frac{1}{2} c_{s}^{2}
\end{array}\right]^{T} .
\end{aligned}
$$

Thus, the stability polynomial associated with the IRKD method is defined by

$$
\varrho(\psi, H)=\operatorname{det}(\psi I-W(H))
$$

where $W(H)$ is given in (61), the characteristic equation is written as follows

$$
\varrho(\psi, H)=q_{0}(H) \psi^{6}+q_{1}(H) \psi^{5}+q_{2}(H) \psi^{4}+q_{3}(H) \psi^{3}+q_{4}(H) \psi^{2}+q_{5}(H) \psi+q_{6}(H) .
$$

\section{NUMERICAL RESULTS}

To illustrate the numerical efficiency of the new IRKD5 method, a set of problems is tested numerically. The new IRKD5 method is compared with the well-known RK methods in the scientific literature. In the numerical comparisons, we used the criterion which is the test based on computing the maximum global error $\left(\log _{10}\right.$ (Max Error)) versus the computational effort measured by $\log _{10}$ (Function Evaluations) required by each problem as demonstrated in Figures 1- 4. The methods chosen in the numerical experiments are as follows:

- IRKD5: The fifth-order IRKD method with four-stage constructed in this paper.

- RK5: The fifth-order Runge-Kutta method given in Dormand et al. [14].
- RKD5: The fifth-order RKD method with three-stage defined by Mechee et al. [6].

Problem 1: The inhomogeneous linear problem given in [15].

$$
y^{\prime \prime \prime}=y+\cos (x), \quad 0<x \leq 1
$$

with initial condition,

$$
y(0)=0, \quad y^{\prime}(0)=0, \quad y^{\prime \prime}(0)=1 .
$$

The exact solution:

$$
y(x)=\frac{1}{2}\left(\mathrm{e}^{x}-\cos (x)-\sin (x)\right) .
$$


Problem 2:

$$
y^{\prime \prime \prime}=-e^{-x}, \quad 0<x \leq 1
$$

with initial condition,

$$
y(0)=1, \quad y^{\prime}(0)=-1, \quad y^{\prime \prime}(0)=1 .
$$

The exact solution: $y(x)=\mathrm{e}^{-x}$.

Problem 3:

$$
y^{\prime \prime \prime}=\left(12 x-8 x^{3}\right) y, \quad 0<x \leq 1
$$

with initial condition,

$$
y(0)=1, y^{\prime}(0)=0, y^{\prime \prime}(0)=-2 .
$$

The exact solution: : $y(x)=\mathrm{e}^{-x^{2}}$.

Problem 4: The nonlinear system

$$
\begin{aligned}
& \mathrm{y}^{\prime \prime \prime}=\mathrm{z}+\frac{1}{\sqrt{\mathrm{u}^{2}+\mathrm{z}^{2}}}-\frac{1}{\sqrt{\mathrm{y}+\mathrm{z}^{2}}} \\
& \mathrm{z}^{\prime \prime \prime}=-\mathrm{y}+\frac{1}{\sqrt{\mathrm{u}^{2}+\mathrm{z}^{2}}}-\frac{1}{\sqrt{\mathrm{y}+\mathrm{z}^{2}}}, \\
& \mathrm{u}^{\prime \prime \prime}=\mathrm{z}+\frac{1}{\sqrt{\mathrm{u}^{2}+\mathrm{z}^{2}}}-\frac{1}{\sqrt{\mathrm{y}+\mathrm{z}^{2}}}, 0<x \leq 1
\end{aligned}
$$

with initial condition,

$$
\begin{array}{ll}
y(0)=1, & y^{\prime}(0)=0, \quad y^{\prime \prime}(0)=-1, \\
z(0)=0, & z^{\prime}(0)=1, \quad z^{\prime \prime}(0)=0, \\
u(0)=1, & u^{\prime}(0)=0, \quad u^{\prime \prime}(0)=-1 .
\end{array}
$$

The exact solution:

$$
y(x)=\cos (x), \quad z(x)=\sin (x), \quad u(x)=\cos (x) .
$$

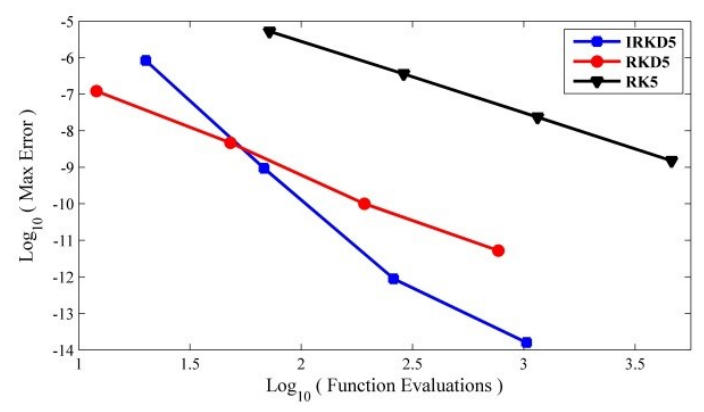

Figure 1. The efficiency graphs for Problem 1 with $h=1 / 2^{i}, \quad i=2,4,6,8$.

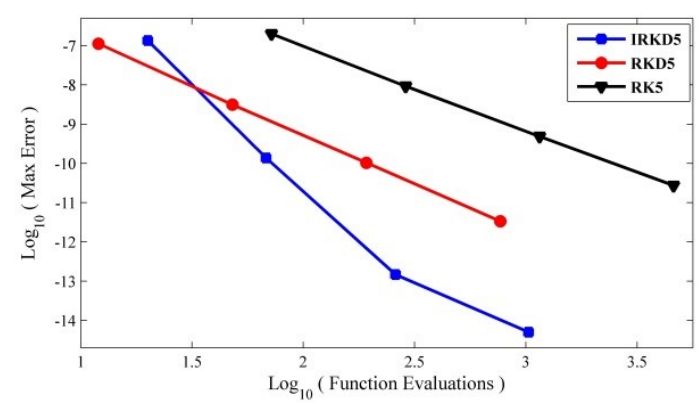

Figure 2. The efficiency graphs for Problem 2 with $h=1 / 2^{i}, \quad i=2,4,6,8$.

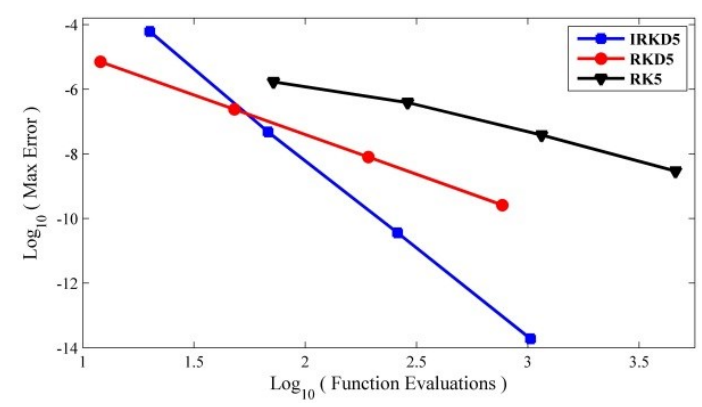

Figure 3. The efficiency graphs for Problem 3 with $h=1 / 2^{i}, \quad i=2,4,6,8$.

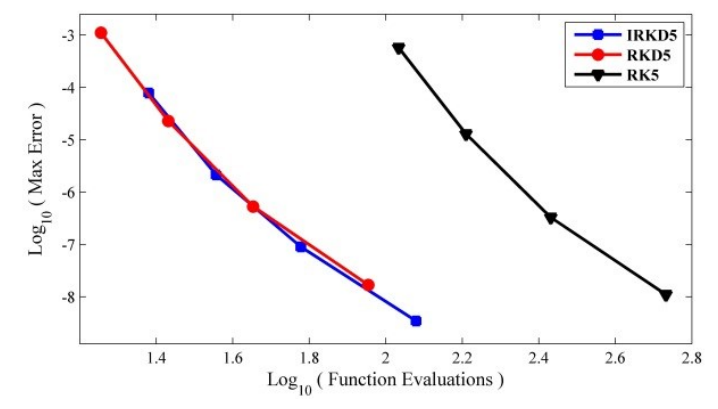

Figure 4. The efficiency graphs for Problem 4 with $h=0.8 / 2^{i}, \quad i=0,1,2,3$. 


\section{CONCLUSION}

An improved Runge-Kutta IRKD method for direct solution of special third-order ODEs is constructed in this paper. The order conditions for the IRKD method were derived and based on the order conditions, we have obtained the fifth-order IRKD method with four-stage, denoted as IRKD5. The stability polynomial of IRKD method is presented. Numerical experiments are performed on several problems and the numerical results show the robustness and competency of the new IRKD5 method when it is compared with the RK5 method and direct RKD5 method in terms of error accuracy and number of function evaluations. For all problems IRKD5 method gives the smallest error for the same number of function evaluations followed by RKD5 and RK5 methods. Hence, it can be concluded that IRKD5 method is the most efficient method compared to RKD5 method and RK5 method in solving special third-order ODEs.

\section{REFERENCE}

1. Awoyemi D. O. (2003). A P-Stable Linear Multistep Method for Solving General ThirdOrder Ordinary Differential Equations. International Journal of Computer Mathematics 80(8), pp. 987-993.

2. Awoyemi D. O. and Idowu O. M. (2005). A Class of Hybrid Collocations Methods for ThirdOrder Ordinary Differential Equations. International Journal of Computer Mathematics 82(10), pp. 1287-1293.

3. Mehrkanoon S. (2011). A Direct Variable Step Block Multistep Method for Solving General Third-Order ODEs. Numerical Algorithms 57 (1), pp. 53-66.

4. Bhrawy A. H. and Abd-Elhameed W. M. (2011). New Algorithm for the Numerical Solutions of Nonlinear Third-Order Differential Equations Using Jacobi-Gauss Collocation Method. Mathematical Problems in Engineering, vol. 2011, Article ID 837218.

5. Waeleh N., Majid Z. A., Ismail F. and Suleiman M. (2012). Numerical Solution of Higher Order Ordinary Differential Equations by Direct Block Code. Journal of Mathematics and Statistics 8(1), pp. 77-81.
6. Mechee M., Senu N., Ismail F., Nikouravan B. and Siri Z. (2013). A Three Stage Fifth-Order Runge-Kutta Method for Directly Solving Special Third-Order Differential Equation with Application to Thin Film Flow Problem. Mathematical Problems in Engineering, vol. 2013, Article ID 795397.

7. Majid Z. A., Azmi N. A., Suleiman M. B. and Ibrahaim Z. B. (2012). Solving Directly General Third Order Ordinary Differential Equations Using Two-Point Four Step Block Method. Sains Malaysiana, 41(5), pp. 623-632.

8. Suleiman M. B. (1989). Solving Nonstiff Higher Order ODEs Directly by The Direct Integration Method. Appl. Math. Comput. 33, pp. 197-219.

9. Rabiei F. (2012). Improved Runge-Kutta Method for Solving Ordinary and Fuzzy Differential Equations. PhD Thesis, Universiti Putra Malaysia.

10. Butcher J. C. (2008). Numerical Methods for Ordinary Differential Equations. Second Edition. John Wiley \& Sons Ltd., England.

11. Lambert J. D. (1991). Numerical Methods for Ordinary Differential Systems, The Initial Value Problem. John Wiley \& Sons Ltd., England.

12. Henrici P. (1962). Discrete Variable Methods in Ordinary Differential Equations. John Wiley \& Sons Ltd., New York.

13. Hairer E., Norsett S. P. and Wanner G. (1993). Solving Ordinary Differential Equations I: Nonstiff Problems. Springer-Verlag, Berlin.

14. Dormand J. R., El-Mikkawy M E. A. and Prince P. J. (1987). Families of Runge-Kutta- Nyström Formulae. IMA J. Numer. Anal. 7, pp. 235-250.

15. You X. and Chen Z. (2013). Direct Integrators of Runge-Kutta Type for Special third-Order Ordinary Differential Equations. Appl. Numer. Math. 74, pp. 128-150. 